\title{
Isolation and identification of Candida Species in Patients with Vulvovaginal Candidiasis
}

\section{Lakshmi Krishnasamy*, Sharanya Krishnakumar, Priya Santharam and Chitralekha Saikumar}

Department of Microbiology, Sree Balaji Medical College and Hospital, Chennai - 600 044, India.

\begin{abstract}
Abnormal vaginal discharge is one of the frequent complaints of women of reproductive age group. This study was carried out to determine the prevalence of vulvovaginal candidiasis(VVC) among the patients attending the tertiary care hospital with complaints of suggestive of vaginitis. This study was done in a tertiary care hospital, Chennai for a period of 1 year from January 2017 to December 2017. The study included 160 women of the age group 15 - 65 years with complaints suggestive of vaginitis. High vaginal swabs were taken and subjected to direct microscopy, cultured onto Sabouraud Dextrose Agar (SDA) and Hichrome Candida differential agar. Candida species were determined by standard microbiological methods and the results were confirmed by automated VITEK2 compact. Candida species were isolated from 56 patients which included C.albicans (25), C.tropicalis (20), C.glabrata (6), C.parapsilosis(4), C.krusei(1). Our study shows higher prevalence of non albicans Candida causing VVC. Hence, we recommend that the investigations up to species identification of Candida may be routinely followed in the microbiology laboratories.
\end{abstract}

Keywords: Candida species, vulvovaginal candidiasis, risk factors.

*Correspondence: laksh45@gmail.com; +91-9944336732

(Received: 11 October 2018; accepted: 30 November 2018)

Citation: Lakshmi Krishnasamy, Sharanya Krishnakumar, Priya Santharam and Chitralekha Saikumar, Isolation and identification of Candida Species in Patients with Vulvovaginal Candidiasis, J Pure Appl Microbiol., 2018; 12(4):2269-2273. http://dx.doi. org/10.22207/JPAM.12.4.67

C The Author(s) 2018. Open Access. This article is distributed under the terms of the Creative Commons Attribution 4.0 International License which permits unrestricted use, sharing, distribution, and reproduction in any medium, provided you give appropriate credit to the original author(s) and the source, provide a link to the Creative Commons license, and indicate if changes were made. 


\section{INTRODUCTION}

Fungal infections have become an alarming problem over the last ten years mainly because of global increase in the number of immunocompromised patients, who are highly susceptible to opportunistic infections, including mycoses ${ }^{1}$. According to a recent study, the incidence of Candidaemia is 6.9 per 1,000 ICU patients of which $7.5 \%$ received antifungal therapy ${ }^{2}$. Among the women, vaginal candidiasis has become a common finding worldwide and up to $75 \%$ of them have symptomatic vaginal candidiasis at least once $^{3}$. Vulvovaginal candidiasis (VVC) is defined as signs and symptoms of inflammation of the vulva and vagina in the presence of Candida spp. and in the absence of other infectious etiology". The clinical features of VVC include pruritus, hyperemia, vaginal discomfort and leucorrhea, burning, soreness, dyspareunia and vaginal or vulvar erythema, which may cause a problem in marital and sexual relations ${ }^{5}$.

Candida albicans appears to be the cause for 80 to 92 percent of vulvovaginal candidiasis 6 . There is an increase in frequency of other candida species nowadays, specifically of $C$. glabrata, may be because of increased use of over-the counter drugs, long-term use of azoles, and the use of short courses of antifungal drugs ${ }^{7}$. The prevalence of candida in India is estimated to be $30 \%$. There are various predisposing factors of VVC, few of which are, hormonal fluctuations in pregnancy, luteal phase of menstrual cycle, use of oral contraceptives, and hormone replacement therapy ${ }^{9}$.

Candida albicans is considered as an important fungal pathogen among humans because of its varying virulence factors that leads to candidiasis like phenotypic switching, phospholipase, proteinase and hemolytic activity. $85-90 \%$ of all cases of vulvovaginal candidiasis shows C.albicans followed by C.glabrata (5-10\%), C. tropicalis (3-5\%) and other species ${ }^{10}$. Though VVC is one of the most common fungal disease worldwide, the information about the distribution and etiology of it is scarcely known to us, because microbiology tests to identify the species of Candida and their antifungal susceptibility testing are not routinely performed in most of the laboratories ${ }^{11}$. Hence, this study is undertaken to determine the species specific prevalence and the risk factors associated with occurrence of VVC.

\section{MATERIALS AND METHODS}

This study was conducted in a tertiary care hospital in Chennai over a period of 1 year. The present study enrolled 160 patients attending the Obstetrics and Gynaecology Out patients department and Inpatients admitted in the wards with symptoms suggestive of vaginitis. The study has been approved by institutional ethical committee and Informed consent was obtained from all the subjects.

Sterile vaginal swabs were used for collection of vaginal samples from the patients. Two high vaginal swab samples were collected aseptically from the posterior vaginal fornix using speculum and posterior vaginal wall retractor. The swabs were transferred to the microbiology laboratory and processed immediately.

One of the swabs was used for Gram stain and direct wet mount microscopy using $10 \%$ Potassium hydroxide solution to determine the presence of yeast cells in the sample. The second swab was streaked on Sabouraud Dextrose agar (SDA) ( HiMedia, India) plates containing chloramphenicol and incubated at $37^{\circ} \mathrm{C}$ for $24-48$ hours. The colonies from SDA plate were subjected to Gram stain to confirm the growth of candida and were further subtyped by streaking on Hichrome Candida differential agar ( HiMedia, India) and incubated at $37^{\circ} \mathrm{C}$ for $24-48$ hours. The pigmented colonies were further examined for assimilation of various sugars and candida species were identified using standard microbiological methods. The colonies from the SDA plate were again processed in automated VITEK2 compact (bioMérieux) and the results were documented.

The colonies from SDA plate were also subjected to germ tube test to check for the production of germ tubes. Germ tube test is done by mixing 3-4 colonies in $0.5 \mathrm{ml}$ human serum and incubated at $37^{\circ} \mathrm{C}$ for $2-4$ hours and examined under microscope for the formation of germ tube. Colonies suggestive of $C$.albicans were confirmed by this germ tube test. 


\section{RESULTS}

A total of 160 women with complaints suggestive of vulvovaginal infections were enrolled in the present study. Their age ranged from $15-65$ years (table -1). In our study 51 women presented with complaints of white discharge (32\%), 45 women (28\%) had complaints of itching and 18 women (11\%) had pain.

Table 1. Agewise distribution of the study subjects

\begin{tabular}{lcc}
\hline $\begin{array}{l}\text { Age in } \\
\text { years }\end{array}$ & $\begin{array}{c}\text { No. of } \\
\text { patients }(\mathrm{n}=160)\end{array}$ & $\begin{array}{c}\text { Percentage } \\
(\%)\end{array}$ \\
\hline$<20$ & 2 & 1.2 \\
$20-40$ & 124 & 77.5 \\
$40-60$ & 31 & 19.4 \\
$>60$ & 3 & 1.9 \\
\hline
\end{tabular}

Table 2. Risk factors associated with the study subjects :

\begin{tabular}{ll}
\hline Risk factor of VVC & No. of patients \\
\hline Pregnancy & 20 \\
Antibiotic usage & 4 \\
Oral contraceptive pills usage & 4 \\
Diabetes & 6 \\
Tuberculosis & 1
\end{tabular}

Out of the 160 high vaginal swab samples, Candida were isolated from 56 samples. The isolated candida was processed for species identification. The Various candida species isolated in the current study were shown in the table.

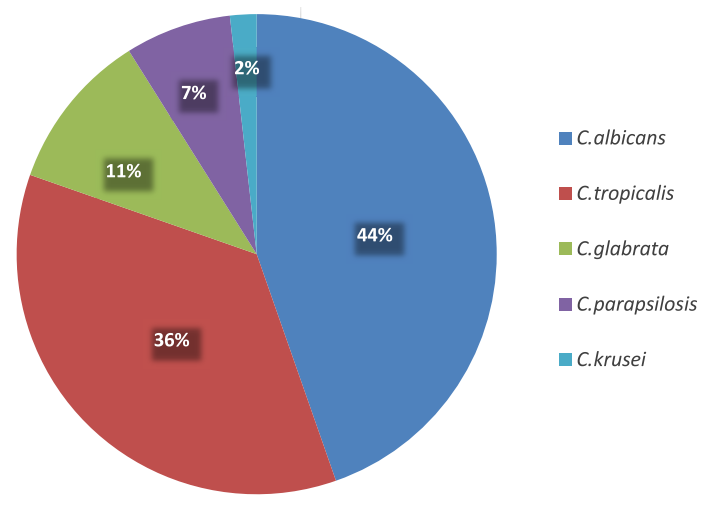

Fig. 1. Percentage wise distribution of various Candida species causing vulvovaginal candidiasis
Table 3. Species distribution of candida

\begin{tabular}{lc}
\hline Candida species & No. of isolates \\
\hline C. albicans & 25 \\
C. tropicalis & 20 \\
C. glabrata & 6 \\
C. parapsilosis & 4 \\
C. krusei & 1 \\
\hline
\end{tabular}

\section{DISCUSSION}

Vaginitis is a universal problem affecting millions of women globally. Vulvovaginal candidiasis (VVC) is defined as signs and symptoms of inflammation of the vulva and vagina in the presence of Candida spp. and in the absence of other infectious etiology ${ }^{12}$.

It is an established fact that C.albicans can convert into a disease causing pathogen from a commensal, when there is a change in the host environment ${ }^{13}$. The increasing antifungal resistance leading to treatment failures and added mortality warrant the need for identification of species in candida. The incidence is more common in women with diabetes where high blood sugars favour growth of candida. Other common associations for the predisposition of candida include pregnancy, antibiotic use and rarely infections like Tuberculosis.

In our study of 160 women, 56 high vaginal swabs $(35 \%)$ were culture positive and grew candida species. This data is similar to reports by Kumari et al (30.6\%), but lower than reports from Namrata Kalia et al (47\%) and ranks second as the cause for vulvo vaginal infections ${ }^{14}$.

Among the 56 women with positive cultures, 20 were pregnant (35\%), 6 women were diabetic (11\%), 4 had a history of antibiotic use (7\%), and 4 were taking Oral contraceptive pills (OCP) (7\%). In our study, there was significant association of positive cultures with pregnancy which could be attributed to high levels of reproductive hormones inducing higher glycogen content in vaginal epithelial cells favouring growth of candida. Some studies say that estrogens have a direct effect on the growth of Candida and its adherence to the vaginal epithelium which explain the increased incidence in women using OCPs ${ }^{15,16}$.

The other major risk factor is diabetes where the likely reasons could be due to 
uncontrolled blood sugars and also the use of unsuitable antifungal agents ${ }^{17}$. The use of antibiotics acts as a short term risk factor for the symptomatic vulvovaginal candidiasis due to the loss of lactobacilli and other normal flora in the vaginal surface ${ }^{18}$.

Most patients who seek medical attention have complaints of white discharge, itching and pain. In our study 51 women had complaints of white discharge (32\%) which was the major complaint. It was followed by complaints of itching which was seen in 45 women $(28 \%)$ and complaints of pain by 18 women (11\%). The clinical presentation was slightly different from reports by Latha ragunathan et $a /^{19}$, where itching (31\%) was the major complaint followed by white discharge (29.4\%) and pain (15.6\%).

In our study identification of candida species was done by conventional methods and confirmed by Vitek 2 compact. There were various candida species detected in our study with highest being C. albicans (44\%), and non albicans (56\%). Our study showed results similar to study by Kumari et $a l^{12}$ and studies from other parts of the world where there was a higher prevalence of non albicans species ${ }^{20,21,22}$. This shows the increasing trend of non albicans species, which could be due to environmental variation. The increasing frequency warrants the need to identify the species and test the anti-fungal susceptibility to commonly used antifungal drugs.

The prevalence of non albicans species in our study were as follows C.tropicalis - 20 (36\%), C.glabrata - $6(11 \%)$, C.parapsilosis - $4(7 \%)$, C.krusei-1 (2\%). Among the NAC (Non albicans Candida) C.tropicalis was the predominant species followed by C.glabrata which was similar to studies by Jayalakshmi et $a^{23}$ and Sundar Khadka et $a^{24}$, but there was a slight difference in the study by Faraji et $a{ }^{17}$ where candida was isolated from diabetic women only. The predominant Non albicans species was C.glabrata followed by C.tropicalis in the study by Sundar Kadka et a ${ }^{24}$. The other species C.parapsilosis, C.krusei have been reported less frequently in patients with vulvovaginitis similar to our study ${ }^{23}$.

Thus, this study shows a changing trend in the causative agents of VVC. Increasing emergence and spread of various non albicans candida is a major concern in the management of VVC.

\section{CONCLUSION}

Candida albicans was found to be the predominant species isolated in the current study followed by C.tropicalis. Yet, there is higher prevalence of non albicans candida species in the study. Hence, screening of all women with vulvovaginal infections for different species of candida would be helpful in providing better care. Thus, complete identification of causative agent of Vulvovaginal candidiasis upto species level in all the microbiology laboratories is highly recommended in order to study the emergence and spread of non albicans candida in the community. More intensive studies are needed to determine the optimal treatment of antifungal drugs for VVC caused by non albicans species.

\section{REFERENCES}

1. Kumari V, Banerjee T, Kumar P, et al. Emergence of non-albicans Candida among candidal vulvovaginitis cases and study of their potential virulence factors, from a tertiary care center, North India. Indian J. Pathol. Microbiol. 2013; 56:144-7.

2. Pahwa N, Kumar R, Nirkhiwale S, et al. Species distribution and drug susceptibility of candida in clinical isolates from a tertiary care centre at Indore. Indian J. Med. Microbiol. 2014; 32:44-8.

3. Latha Ragunathan, G.K Poongothai, Annie Rofeena Sinazer, et al. Phenotypic characterization and antifungal susceptibility pattern to fluconazole in candida species isolated from vulvovaginal candidiasis in a tertiary care hospital. Journal of Clinical and Diagnostic Research. 2014 ; 5: DC01 - DC04

4. Achkar JM, Fries BC. Candida infections of the genitourinary tract. ClinMicrobiol Rev. 2010; 23:253-73.

5. Moreira D, Paula CR. Vulvovaginal candidiasis. Int. J .Gynaecol Obstet. 2006; 92:266-7.

6. Odds, FC. Candidosis of the genitalia. In: Odds, FC. Candida and candidosis: A review and bibliography, 2nd ed, Bailliére Tindall, London 1988, p. 124.

7. Horowitz BJ, Giaquinta D, Ito S. Evolving pathogens in vulvovaginal candidiasis: implications for patient care. J. Clin Pharmacol. 1992; 32:248-55.

8. Thulkar, J., Kriplani, A., Agarwal, N., et al. Aetiology \& risk factors of recurrent vaginitis $\&$ its association with variouscontraceptive methods. Indian J.Med. Res. 2010; 131: 8387.

9. Geiger AM, Foxman B, Gillespie BW. The 
epidemiology of Vulvovaginal candidiasis among university students Am. J. Public Health.1995; 85:1146-8.

10. Peters RB, Bahn AN, Barens G. Candida albicans in the oral cavities of diabetics. J Dent. Res. 1966; 45:771-777

11. Paulitsch A, Weger W, Ginter-Hanselmayer G, et al. A 5-year (2000-2004) epidemiological survey of Candida and non-Candida yeast species causing vulvovaginal candidiasis in Graz, Austria. Mycoses 2006; 49:471-5.

12. Kumari V, Banerjee T, Kumar $\mathrm{P}$, et al. Emergence of non-albicans Candida among candidal vulvovaginitis cases and study of their potential virulence factors, from a tertiary care center, North India. Indian J. Pathol Microbiol. 2013; 56:144-7

13. Sharma Y, Chumber SK, Kaur M. Studying the prevalence, species distribution, and detection of in vitro production of phospholipase from Candida isolated from cases of invasive candidiasis. J. Global Infect Dis. 2017; 9:8-11

14. Namarta Kalia, Jatinder Singh, Sujata Sharma, Sukhdev Singh Kamboj, Hardesh Arora, Manpreet Kaur. Prevalence of Vulvovaginal Infections and Species Specific Distribution of Vulvovaginal Candidiasis in Married Women of North India. IJCMAS. 2015; 4(8):253-266

15. Drake TE, Maibach HI, Candida and candidiasis: cultural conditions, epidemiology, and pathogenesis. Postgrad Med. 1973; 53:83-7.

16. Atousa Aminzadeh, Ali Sabeti Sanat, and Saeed Nik Akhtar. Frequency of Candidiasis and Colonization of Candida albicans in Relation to Oral Contraceptive Pills. Iran Red Crescent Med J. 2016 October; 18(10):e38909.

17. Reza Faraji, Mehr Ali Rahimi, Fatemeh
Rezvanmadani, et al. Prevalence of vaginal candidiasis infection in diabetic women. African Journal of Microbiology Research. 6(11): 27732778

18. Ahmad A, Khan AU. Prevalence of Candida species and potential risk factors for vulvovaginal candidiasis in Aligarh, India. Eur J Obstet Gynecol Reprod Biol. 2009; 144(1):68-71.

19. Latha Ragunathan, G.K Poongothai, Annie Rofeena Sinazer, et al. Phenotypic Characterization and Antifungal Susceptibility Pattern to Fluconazole in Candida species Isolated from Vulvovaginal Candidiasis in a Tertiary Care Hospital. Journal of Clinical and Diagnostic Research. 2014; 5: DC01 - DC04

20. Méan M, Marchetti O, Calandra T. Bench to bedside review: Candida infections in the intensive care unit. Crit Care. 2008; 12:204.

21. Mokaddas EM, Al Sweih NA, Khan ZU. Species distribution and antifungal susceptibility of Candida bloodstream isolates in Kuwait: A 10 year study. J. Med. Microbiol. 2007; 56:255 9.

22. Jain N, Mathur P, Misra MC, et al. Rapid identification of yeast isolates from clinical specimens in critically ill trauma ICU patients. J. Lab. Physicians. 2012; 4:30 4.

23. Jayalakshmi L, RatnaKumari G, Samson SH. Isolation, Speciation and Antifungal Susceptibility Testing of Candida from Clinical Specimens at a Tertiary Care Hospital. Sch. J. App. Med. Sci., 2014; 2(6E):3193-3198

24. Sundar Khadka, Jeevan Bahadur Sherchand, Bharat Mani Pokhrel, et al. Isolation, speciation and antifungal susceptibility testing of Candida isolates from various clinical specimens at a tertiary care hospital, Nepal. BMC Res Notes. 2017; 10:218 